\title{
RECENSIÓN A PÉREZ VALLEJO, A. Ma Y PÉREZ FERRER, F., BULLYING, CIBERBULLYING Y ACOSO CON ELEMENTOS SEXUALES: DESDE LA PREVENCIÓN A LA REPARACIÓN DEL DAÑO, DYKINSON, S.L., MADRID, 2016, 250 PÁGINAS
}

\author{
ALBA PAÑOS PÉREZ \\ Profesora Ayudante Doctora de Derecho Civil \\ Universidad de Almería \\ Email: app806@ual.es
}

La obra objeto de recensión se enmarca en el Proyecto I+D+I, del Programa Estatal de Investigación, Desarrollo e Innovación Orientada a los Retos de la Sociedad: Análisis jurídico y cuantitativo de la violencia en la infancia y adolescencia: propuestas de intervención socio-legal (DER2014-58084), cuyo investigador principal es Ana María Pérez Vallejo, y en el Proyecto de Investigación Variables para una moderna política criminal, superadora de la contradicción expansionista/reduccionista de la pena de prisión (DER 2012-35860), cuyo investigador principal es Lorenzo Morillas Cueva; contando ambos proyecto con la Prof. Fátima Pérez Ferrer como investigador colaborador.

El libro que tengo entre mis manos, publicado por la editorial Dykinson, en su colección "Monografías de Derecho Penal", es resultado del fructífero esfuerzo llevado a cabo durante los últimos años por las autoras Pérez Vallejo y Pérez Ferrer, respaldadas por el equipo de profesores e investigadores de distintas universidades españolas que conforman los Proyectos de Investigación de los que son parte, que han dedicado su tiempo y su actividad científica al análisis, estudio y valoración de un tema de hondo calado social e indudable trascendencia jurídica como es el acoso escolar. 
El lector que se sumerja en esta obra puede apreciar $a b$ initio, desde una perspectiva multidisciplinar, la exhaustividad y el exquisito rigor que contienen sus más de doscientas páginas. Junto a la virtud de la oportunidad y el acierto en la elección del tema, de máxima actualidad por la creciente preocupación social al respecto, la cobertura y difusión por parte de los medios de comunicación y las últimas reformas legislativas en la materia; esta monografía presenta la peculiaridad de que el tema planteado se aborda desde dos disciplinas distintas, indisolubles en este caso, el Derecho Penal y el Derecho Civil, convirtiéndose en un instrumento imprescindible para aquéllos que quieran profundizar sobre la materia.

Realizar la recensión de un libro de estas características es ardua tarea; sin embargo, cuando la obra en cuestión recoge un estudio completo de los planteamientos más actuales sobre la materia, aportando suficientes elementos de juicio e ilustrada con los casos jurisprudenciales de mayor relevancia hasta la fecha de su publicación, el camino se facilita considerablemente. Y es que, con total seguridad, la mejor recensión que se puede hacer de este trabajo es invitar a todo aquel que pretenda reflexionar sobre este tema a que se haga con él y compruebe la certeza de la afirmación realizada; sin duda, le auguro una hacedera lectura gracias a la habilidad de las autoras. Me atrevería a afirmar, sin género de duda, que el rigor y las transversalidad de la investigación realizada hace que ésta sea una obra de referencia y de gran utilidad para los estudiosos y profesionales que deseen profundizar en este ámbito del Derecho Penal y del Derecho Civil.

Baste leer la introducción, de apenas dos páginas, para tomar consciencia de la dimensión que actualmente alcanza del fenómeno del acoso escolar (bullying, acuñado de la extendida terminología anglosajona), una lacra de la sociedad en general y de los Centros educativos en particular, cuya manifestación más gravosa, conductas de acoso y violencia interpersonal, implica exclusión social, intimidación y maltrato infantil. No obstante esta actual preocupación social, académica e institucional por dicho fenómeno, el bullying se ha caracterizado hasta hace muy poco por permanecer oculto.

Así, se ha considerado tradicionalmente algo inevitable y consustancial a las relaciones entre menores (dentro y fuera de los centros educativos), que se toleraba banalizándolo con la socorrida expresión "son cosas de niños", bajo la creencia de que 
se trataba de un problema estrictamente privado que debía ser solventado entre iguales, ajeno por supuesto al ámbito de intervención del sistema legal. Esta tradicional aceptación del acoso escolar, cuando no su negación, han contribuido a que disfrutase de una total impunidad. En este contexto, tal y como ponen de manifiesto las autoras desde el comienzo del estudio, el silencio de las víctimas y los testigos, incluso de los propios centros escolares, ha fomentado el desconocimiento y la minimización del problema.

Hoy día está asumido que el acoso escolar es una realidad presente en la vida de menores y adolescentes, con el agravante de que los medios y tecnologías de la información y comunicación (TICs) pueden ampliar sus efectos devastadores (ciberbullying). Y es que la relación entre las TICs y los menores es cada vez más compleja; considerando los riesgos que comportan actualmente las nuevas tecnologías en las telecomunicaciones y los nuevos medios de difusión, como las redes sociales o los servicios de mensajería instantánea a través de la telefonía móvil, para los menores de edad. Baste aportar el dato de que, a día de hoy, uno de cada cuatro casos de acoso ya se producen vía redes sociales o teléfono móvil; medios que extienden el acoso a las víctimas a entornos ajenos al escolar, las 24 horas del día. El riesgo crece con el avance de la sociedad de la información y las tecnologías y, en este entorno, caracterizado por la inmediatez, la rapidez y la amplia difusión de dichos contenidos, se facilita el ataque a los derechos fundamentales de los usuarios más vulnerables, los menores de edad.

Por otra parte, las redes sociales han invadido nuestra vida y entrañan unos peligros jurídicos que se incrementan alarmantemente respecto a los menores, los cuales se familiarizan con un uso desinhibido e irresponsable de las mismas a edades cada vez más tempranas. No es de extrañar que, en este contexto, se haya acuñado el término "nativos digitales" para aquéllos menores de entre 3 y 11 años que han nacido y desarrollan su personalidad familiarizados de forma natural con un entorno digital. Según la Comisión Europea, 50\% de los menores de 12 años tiene perfil en las redes sociales y la edad de comienzo a navegar por Internet es de 7 años; desde entonces, su uso se va incrementando progresivamente. Si ha esto le sumamos la poca vigilancia por parte de los progenitores, ingenuos o desconocedores de una realidad amenazante, 
puede apreciarse el creciente uso de las redes sociales para ejercer acoso o violencia entre iguales.

A mayor abundamiento, los medios de comunicación muestran un reciente interés por este fenómeno y se hacen eco a diario de noticias relacionadas con el mismo, a raíz de los últimos casos desgraciadamente más mediáticos. De hecho, hace unos meses protagonizó un debate de opinión pública un programa que la cadena Cuatro tenía previsto emitir a finales del pasado mes de junio, Proyecto Bullying, pero que finalmente fue paralizado a instancia de las Fiscalías de Menores correspondientes, por considerar que perjudicaba el interés superior de los menores que aparecían en el mismo (por ser éstos perfectamente identificables). Dicho programa abordaba el tema del acoso escolar, recogiendo testimonios tanto de menores protagonistas del acoso como de personas afectadas de su entorno, con la intención de denunciar y concienciar a la sociedad sobre este problema.

Por lo que se refiere a la estructura formal de la obra, y en relación a los objetivos que las autoras plantean en su elaboración, esto es, la realización de un diagnóstico de la situación actual sobre las conductas de acoso escolar y ciberacoso que permita evaluar las carencias y necesidades detectadas, así como revisar los recursos existentes en la actualidad y plantear propuestas de intervención socio-legal; el libro se divide en dos partes claramente diferenciadas, la referente a la responsabilidad penal y la que contiene el régimen de la responsabilidad civil. Se estructura en cuatro capítulos, en los que abordan sistemáticamente la problemática del acoso escolar desde el ámbito penal hasta el civil, introduciendo al lector en el estudio a través de los aspectos generales del acoso escolar y terminándolo con unas valoraciones finales, que culminan la profunda investigación realizada.

El primero de los capítulos, el más genérico e introductorio, está dedicado a analizar los instrumentos de prevención, pieza clave en el tema objeto de estudio. Las autoras nos sitúan en la gravedad del problema aportando datos de los últimos estudios nacionales y europeos en la materia. Es alarmante descubrir que, según el Informe Cisneros VII (2006), uno de cada cuatro escolares sufre acaso y que es más acusado entre los más pequeños de edad (7 y 10 años), cuando los menores de 14 años son inimputables penalmente. Ante esta realidad, y teniendo en cuenta que la judicialización 
del acoso escolar va en aumento, lo que revela una mayor concienciación de la problemática, se plantea ya desde el inicio la prevención como solución para erradicar el problema.

No obstante, uno de los principales escollos con los que nos encontramos es con el silencio que alienta a los acosadores desde distintos frentes: el silencio de las víctimas, el silencio de los testigos (espectadores u observadores), el silencio de los propios padres y, para mayor frustración, el silencio, la negación o posicionamiento del Centro escolar, que hacen aún más difícil si cabe esta labor preventiva. Por ello, coincido con las autoras al afirmar que en muchos casos el acoso escolar se mantiene por la pasividad y la ignorancia de las personas que rodean tanto a la víctima como a los agresores; actitudes omisivas que pueden obedecer a distintos motivos, analizados en este capítulo con detenimiento. Sobre todos estos frentes será necesario focalizar la prevención.

En este sentido, enfocan el estudio sobre la prevención haciendo especial referencia a los Centros educativos, ya que, tal y como ponen de manifiesto, la complejidad del acoso escolar exige que la actuación ante este tipo de maltrato presente en la infancia y adolescencia, adopte un carácter esencialmente preventivo-educativo. Las actividades formativas sobre educación en valores, educación en Resolución de Conflictos y la Mediación, resultan prioritarias tanto para docentes, padres y menores o adolescentes; así como su implementación uniforme en los Centros educativos de las distintas Comunidades Autónomas. Las autoras nos presentan estos mecanismos como instrumentos especialmente aptos para evitar el acoso escolar y minimizar sus consecuencias en sus primeras fases.

Así, diversos estudios en la materia revelan, pues, que es necesaria una cierta dosis de implicación o colaboración de padres, Centros educativos y sociedad en general; es decir, una intervención simultánea, como única vía posible de prevención del acoso escolar. En este sentido, afirman que el abordaje del acoso ha de ser global ya que produce efectos negativos para todas las partes implicadas, incluido el propio Centro. Algunos estudios proponen sugerencias prácticas para la prevención que paulatinamente se van implementando en algunos Centros educativos y que son recogidas por las autoras. De hecho, la Memoria de la Fiscalía General de Estado (2013) refiere un 
"continuo descenso" de los casos de acoso escolar gracias a las actuaciones preventivas de los colegios y a su vigilancia en el ámbito escolar. Este órgano del Ministerio Fiscal está especialmente involucrado, como no podía ser de otra manera, en la lucha contra el acoso escolar; así, la Instrucción 10/2005, de 6 de octubre, de la Fiscalía General del Estado sobre el tratamiento del acoso escolar desde el sistema de justicia juvenil sirve de guía a las autoras para definir y situar el fenómeno, y será un referente destacado en sus fuentes a lo largo de toda la obra.

Una aportación fundamental en este capítulo es el análisis de la prevención desde tres niveles distintos. La prevención primaria, una primera fase en la que la educación en valores, la información, la sensibilización y consiguiente toma de conciencia del problema del acoso e intimidación entre iguales son las bases; y en la que la responsabilidad recae sobre los padres, que deben abogar por una educación antiviolenta, democrática y no autoritaria, transmitiendo a los hijos valores de convivencia pacífica, responsabilidad y solidaridad. Los colegios, por su parte, son garantes de mejorar la convivencia en sus Centros, prevenir la conflictividad y evitar la aparición de conductas violentas entre iguales, que pudieran desembocar en acoso escolar. Es esencial, en este ámbito, la información que debe suministrarse a los alumnos sobre las implicaciones legales que pueden tener las conductas de acoso y ciberacoso. Y, por otro lado, se sitúa también el foco de prevención en los medios de comunicación, cada vez más interesados por el tema, como ya hemos señalado. Se revelan como un medio útil para la sensibilización y concienciación social frente al fenómeno, aunque se critica que, en ocasiones, generan alarma social y no difunden las noticias con exactitud, tentados más por el morbo que provoca cualquier noticia en la que hay menores implicados. Por ello, es necesario reclamarles desde las instituciones una mayor responsabilidad, ya que les corresponde un deber ético y moral de autorregulación y compromiso con la erradicación del problema.

Una segunda fase de prevención secundaria, en la que entrarían en juego medidas a adoptar (plan de actuación) una vez detectada, en sus inicios, la situación de acoso. Para ello, se señalan algunos de los muchos indicadores que puedan ayudar a detectar que un menor está sufriendo acoso en el colegio. Para evitar llegar a los extremos de agravamiento de la situación de acoso, es fundamental una intervención 
rápida y efectiva con la colaboración y cooperación de todas las partes implicadas: alumnos, padres, profesores y la dirección del centro; y así lo ponen de manifiesto las autoras.

Y una tercera fase de prevención terciaria, correspondiente a las medidas de ayuda a los protagonistas directos de los casos de acoso escolar (víctimas, acosadores y testigos). Este nivel trata de minimizar el impacto en situaciones de acoso ya consolidadas, en el que se utilizan técnicas terapéuticas, de soporte y protección a las víctimas y de soporte y control a los agresores.

Fiel relejo de la actualidad de esta obra es la especial atención a los avances legislativos en la materia, tales como la Ley Orgánica 8/2013, de 9 de diciembre, de Mejora de la Calidad Educativa (LOMCE), que incluye expresamente el fenómeno del acoso escolar; o Ley 26/2015, de 28 de julio, de modificación del sistema de protección a la infancia y a la adolescencia, que modifica la Ley Orgánica 1/1996, de 15 de enero, de Protección Jurídica del Menor (LORPM), que incluye deberes del menor en el ámbito familiar, escolar y social. Más recientemente, el pasado 22 de enero de 2016, el Consejo de Ministros analizó el Borrador del Plan Estratégico de Convivencia Escolar; algunas de las medidas que prevé, como la puesta en marcha de un teléfono gratuito contra el acoso escolar, comenzarán a implantarse en el presente curso 2016/2017.

Por otra parte, resulta muy oportuna, a mi juicio, la inclusión en este capítulo de los planes de convivencia y protocolos de actuación ante supuestos de acoso escolar. Las autoras se centran en la normativa aprobada en la Comunidad Autónoma de Andalucía sobre las medidas y procedimientos dirigidos a realizar actuaciones para la identificación, intervención y seguimiento de la situación de acoso escolar; otra pretensión habría rebasado con creces el objeto de estudio de esta obra. En este sentido, resulta muy interesante la inclusión in fine del Protocolo de Actuación previsto por la Orden de 20 de junio de 2011, reproducido literalmente por resultar altamente estructurado y muy formativo.

Este primer capítulo termina, como no podía ser de otra manera conociendo la dilatada trayectoria en la materia de las Prof. Pérez Vallejo y Pérez Ferrer, fruto de años de investigación y experiencia, con un análisis de la mediación en contextos educativos 
como estrategia de prevención. Tema no exento de polémica por la disparidad de opiniones al respecto; la mediación se presenta, en el ámbito escolar, como un recurso útil y eficaz, particularmente, para la prevención y la resolución de conflictos cotidianos que pueden producirse entre miembros de la comunidad escolar. No obstante, a pesar de estar prevista por la LORPM y de que las autoras consideran altamente positivo un programa de mediación entre compañeros en el ámbito escolar, en el que los alumnos actúen como mediadores; dadas las características especiales del bullying, su eficacia se pone en entredicho. Y es que la mediación requiere que exista igualdad entre las partes implicadas, por lo que para que los conflictos en los que existe una gran diferencia de poder sean mediables sería necesario que desapareciese la misma, proceso que lleva un tiempo.

El segundo capítulo lo dedican a la responsabilidad penal derivada del acoso escolar. Y, a pesar de que ab initio se pone de manifiesto lo contrario (haciendo gala de la modestia que caracteriza a las autoras), lo cierto es que las páginas siguientes son fiel reflejo de una reflexión seria y pormenorizada sobre la problemática del acoso escolar y sus consecuencias en el ordenamiento jurídico penal. Así, se examinan detenidamente sus elementos o presupuestos identificadores y los tipos penal en los que, con mayor frecuencia, estos comportamientos violentos pueden encontrar una adecuada ubicación.

Respecto a los tipos delictivos, destacar que el pasado 1 de julio de 2015 entró en vigor la LO 1/2015, de 30 de marzo, por la que se modifica la LO 10/1995, de 23 de noviembre, del Código Penal y, a pesar de la supresión del Título III “ De las faltas”, se mantienen las conductas de acoso escolar, como delito de lesiones (artículo 147.1 CP) y calificándose como "delito leve" las lesiones de menor entidad (artículo147.2 CP). Pues bien, analizando la infracción penal que, de forma más clara, contiene los elementos que definen las conductas subsumibles en la figura del acoso escolar, nos encontramos ante los delitos contra la integridad moral, previstos y penados en el artículo 173.1 del Código Penal. Destaca que su previsión ha sido calificada de absolutamente novedosa, señalándose su justificación como respuesta adecuada a la necesidad de evitar tratamientos inhumanos o degradantes en el ámbito privado.

El elenco de resoluciones jurisprudenciales analizadas por las autoras pone de manifiesto todas las posibles conductas de hostigamiento, diferenciando entre el acoso 
directo y el acoso indirecto, facilitando así enormemente al lector la comprensión de las distintas manifestaciones del acoso escolar. En consecuencia, éste podría ser penado conforme a otros tipos delictivos, tales como el delito de lesiones (físicas y psíquicas) del artículo $147 \mathrm{CP}$, o en los casos más graves, el delito de inducción al suicidio del artículo 143.1 CP; tipos que, en ocasiones, pueden entrar en concurso con el mencionado artículo 173.1 CP. Y es que, los casos más graves y prolongados de acoso escolar pueden desembocar en el suicidio de los menores acosados, aunque no será nada fácil mantener una acusación y fundamentar una sentencia condenatoria por este tipo delictivo, demostrando la relación de causalidad entre los actos de acoso y el resultado de muerte. Desgraciadamente, tal y como se pone de manifiesto, los adolescentes que sufren acoso escolar tiene mayor propensión al suicidio. Por otra parte, la modificación realmente relevante a efectos del análisis del acoso escolar, es la que se produce con la tipificación del delito de acoso del artículo 172 ter $\mathrm{CP}$, introducido ex novo en virtud de lo dispuesto en el artículo 91 de la LO 1/2015, y que conllevará que muchas de las conductas hasta ahora consideradas constitutivas de bullying puedan castigarse por la vía del nuevo artículo 172 ter $\mathrm{CP}$.

De forma pormenorizada se analiza la responsabilidad penal del menor infractor y las medidas a adoptar conforme a la Ley Orgánica reguladora de la Responsabilidad Penal del Menor, incidiendo en que los menores de 14 años son inimputables penalmente, por lo que existe una franja importante de alumnos menores de esa edad a los que no se les exigirá responsabilidad penal. En este sentido, puede afirmarse que, cuando las conductas de acoso cometido por alumnos menores (de entre 14 y 18 años) son penalmente típicas conforme a los delitos anteriormente expuestos, además de la responsabilidad penal, habrá una responsabilidad civil derivada del ilícito penal, cuya regulación cuenta con disposiciones específicas en la propia Ley Penal del Menor (artículos 61 a 64 LORPM).

Nuevamente, este capítulo culmina con un excursus sobre la mediación en el sistema de justicia penal juvenil, en el que se deja constancia de que la propia LORPM articula la posibilidad de una mediación con menores infractores sobre el reconocimiento expreso del principio de oportunidad en el ejercicio de la acción penal, así como del superior interés del menor y del principio de proporcionalidad. Sin obviar 
las indudables ventajas de este método, habrá que atenerse a las limitaciones ya aducidos anteriormente.

El tercer capítulo completa con maestría la perspectiva penal abordada en la obra al analizar otras formas de acoso entre iguales con elementos sexuales: el delito de child grooming y sexting. El título VIII del Libro II del vigente Código Penal, bajo la rúbrica "Delitos contra la libertad e indemnidad sexual", ha sido objeto en los últimos años de una prolija y dinámica actividad legislativa, constituyendo, de este modo, una de las materias más significativas y complejas en su previsión en el ordenamiento jurídicopenal español. En este capítulo se aborda, desde una perspectiva jurídico-penal, la problemática del delito denominado child grooming y del delito de "embaucamiento", los cuales incriminan conductas que, desgraciadamente, cada día aumentan vertiginosamente, favorecidas por la aparente impunidad que facilita el anonimato de las últimas tecnologías.

Nos adentramos con el capítulo cuarto en la parte de la obra dedicada a la responsabilidad civil y la reparación del daño por bullying, ciberbullying y acoso con elementos sexuales. Conductas antijurídicas que, además del reproche criminal que merecen, conforme a la legislación penal del menor ya expuesta, producen un daño efectivo que nadie tiene obligación de soportar, dando lugar al nacimiento de la correspondiente responsabilidad civil, donde estarán implicados uno o varios menores (agresores y víctimas), sus progenitores o representantes legales y guardadores.

Pues bien, a pesar de la profundidad del tema y de las enormes dificultades que conlleva su investigación, por la complejidad de los diferentes regímenes jurídicos aplicables y los órdenes jurisdiccionales distintos para su enjuiciamiento (civil, contencioso y jurisdicción de menores); las autoras se desenvuelven con extraordinaria soltura en el análisis del régimen que disciplina dicha responsabilidad civil derivada del acoso escolar. Distinguirán, en función del tramo de edad del menor y de si la legislación a aplicar es la civil (menores de catorce años) o la derivada de la LORPM (14 a 18 años). Diferencias sustantivas y procesales que se reflejan también, como se adelanta, en función de si el hecho ilícito causante del daño está o no tipificado penalmente (ilícito penal o ilícito civil). 
Es sin duda llamativa, por tanto, la claridad sobresaliente en la exposición de este entramado legal y judicial que, como vemos, caracteriza el ámbito de la responsabilidad civil derivada del acoso escolar, en el que coexisten una pluralidad de regímenes jurídicos “injustificables”, en palabras de las autoras, que disciplinan una misma realidad jurídica en materia de Derecho de daños. Contribuye a clarificar la cuestión las soluciones adoptadas por los Tribunales, que aportan a lo largo del desarrollo del tema.

Como adelantábamos, cuando los autores de actos o conductas de acoso escolar (estén o no tipificadas penalmente) son menores de 14 años, así como cuando los hechos no revistan entidad para calificarse de ilícito penal, pero sí civil y sus autores cuenten entre 14 y 18 años, se aplicarán las reglas básicas de la responsabilidad civil (artículos 1902 y $1903 \mathrm{CC}$ ). A pesar de que las autoras ponen de manifiesto que difícilmente un menor de 14 años puede tener "capacidad de culpa civil" (que sería presupuesto para su responsabilidad directa por los daños causados por el acoso), debo disentir al respecto. Dada la precoz iniciación en el uso de Internet por parte los menores, actualmente se podría rebajar la edad de madurez legal civil hasta los 12 años (límite establecido incluso en el CC para tener en cuenta a los menores en determinadas circunstancias); edad a la que ya podrían ser conscientes de sus propios actos y de las repercusiones de los mismos, teniendo en cuenta además que, tal y como hemos señalado, el acoso escolar es más acusado entre los menores de entre 7 y 10 años de edad. La acción de responsabilidad civil se entablará bien ante la jurisdicción civil, cuando se demande a los padres del acosador (culpa in educando) o al Centro docente privado (culpa in vigilando) o a ambos a la vea; o bien, ante la jurisdicción contenciosa cuando el menor está bajo la guarda de la Administración Pública (Centro docente público).

Por otra parte, cuando se trata de mayores de 14 años y menores de 18, además de la responsabilidad penal y civil que les es exigible, hay otros responsables civiles que responderán solidariamente con él ante la jurisdicción de menores, tal y como lo establece la LORPM, en sus artículos 61.3 y 4. Para clarificar la cuestión, las autoras recurren a la amplia casuística judicial, que refleja cómo en unos casos se demanda a los padres de los menores autores de las conductas de acoso y, en otros, al Centro docente; 
reparando en el distinto orden jurisdiccional que conoce de los hechos (civil o contencioso) y en el derecho sustantivo aplicable, que no siempre es uniforme. La introducción de este régimen de responsabilidad solidaria con el menor responsable de los hechos de sus padres, tutores, acogedores o guardadores, se considera "revolucionario", si bien al permitir la moderación judicial la objetivación de esta responsabilidad se torna incierta.

Son interesantes, asimismo, las páginas que el libro dedica, a continuación, a los responsables civiles antes la jurisdicción de menores por los delitos de child grooming y sexting. Y, por último, mención especial en este capítulo merece la reparación del daño por las distintas modalidades de acoso estudiadas a la largo de toda la obra. Que las viejas y nuevas formas de acoso en menores y adolescentes producen daños en su joven personalidad en formación no admite matices; siendo independiente que el daño provenga de un ilícito penal o de un ilícito civil. Ahora, especial atención requiere determinar qué daños son resarcibles o compensables por estas conductas; ya que si es incuestionable que los daños materiales y patrimoniales son fácilmente resarcibles, éstos raramente concurren en las conductas de acoso, en las que suelen existir daños físicos o psíquicos y, particularmente, daños morales.

Así pues, en todas las sentencias que condenan por conductas de bullying, ciberbullying, child grooming o sexting, la indemnización viene referida al daño moral sufrido por el menor víctima. En este sentido, se apunta que la vulneración de estos bienes y derechos, desde luego intangibles, no sería susceptible de indemnización en sentido estricto, sino de compensación; resultando muy complicada la cuantificación de la misma. En consecuencia, y con excelente criterio, las autoras culminan este capítulo con un apartado dedicado al llamado "precio del dolor", en el que dan respuesta al interrogante “¿cuánto vale el dolor, padecimiento o aflicción de un niño o adolescente víctima?” a través de un exhaustivo análisis de jurisprudencia realizado para visualizar su cuantía e importe.

Por el interés y la profundidad del tema abordado, y porque recoge propuestas de futuro junto con las reflexiones de las autoras después del arduo trabajo de investigación realizado, esta obra no podía terminar sino con unas valoraciones personales. En ellas se reitera que la preocupación e interés por el tema se ha visto incrementada por la 
repercusión mediática que han tenido algunas situaciones graves y por las consecuencias tan devastadoras que el acoso puede producir, fundamentalmente con el auge de las TICs. Frente a ello, consideran que la creación de un consenso moral sobre la importancia de la dignidad de las personas que confluyen en un centro escolar es el mejor antídoto contra una oleada de violencia dentro de las escuelas, con todas las consecuencias negativas que esto puede originar. Así, tal y como se pone de manifiesto a lo largo del libro, la mejor forma de prevenir este fenómeno es la educación y la construcción de una convivencia igualitaria, estructurada a través de una simultaneidad de acciones y en distintos niveles.

Como consecuencia de todo lo anterior, y tras la detenida lectura de la obra, debo concluir que la prevención es la clave sobre la que hay que focalizar los esfuerzos para la erradicación de esta lacra social que afecta a las personas más vulnerables, los menores, con derecho a desarrollarse en un ambiente de paz y seguridad en los Centros educativos. Es esencial que se implemente la prevención de la violencia escolar en los Centros educativos a través de una doble línea de actuación formativa e informativa, que ayude a concienciar y persuadir a los menores (potenciales agresores y testigos) sobre las graves consecuencias de estas conductas. En este sentido, sería conveniente unificar los Protocolos de Actuación de los Centros de toda España, para consensuar las medidas que deben adoptar los mismos respecto a la identificación, intervención y seguimiento de la situación de acoso escolar.

Por tanto, se debe exigir mayor concienciación e implicación a los Centros docentes, ya que la comunidad escolar es la más capacitada para resolver el conflicto y el entorno idóneo para tratarlo. Esta necesaria creación de entornos vitales de calidad en el contexto educativo no debe olvidar el fundamental papel de la familia para la educación en valores. Solamente en caso de que las medidas preventivas no funcionasen, entraría en juego el sistema judicial; teniendo presente el principio de subsidiariedad que configura el papel subsidiario y reactivo de la justicia juvenil, según el cual el Derecho Penal debe ser la última ratio. Así, las líneas de política-criminal en esta materia deben primar la estrategia preventiva sobre la punitiva o represiva.

Se trata, pues, de un magnífico trabajo cuya lectura procura una interesante y pormenorizada información sobre su objeto, sugiriendo al lector interrogantes sobre un 
tema de máxima actualidad que preocupa a toda la sociedad y que hace tambalear los cimientos del Estado Social y Democrático de Derecho, caracterizado por los valores democráticos de diálogo, respeto y tolerancia. Aun así, las autoras concluyen con una perspectiva optimista al enfatizar in fine que, en nuestro país, aunque es mucho más el camino que nos queda por recorrer para erradicar totalmente esta dramática situación, las líneas de intervención que se han seguido en los últimos años han logrado detener, en parte, el avance del problema, e incluso obtener algunos éxitos parciales al lograr disminuciones significativas en determinadas conductas de acoso. A la lucha contra este mal contribuyen especialmente iniciativas como este estudio, respaldado por dos Proyectos de Investigación y materializado en la presente obra, que he tenido el honor de someter a análisis. 